\title{
An automatic recognition and parameter extraction method for structural planes in borehole image
}

\author{
Chuanying Wang ${ }^{1 *}$, Xianjian Zou ${ }^{1,2}$, Zengqiang Han ${ }^{1}$, Yiteng Wang ${ }^{1}$, Jinchao Wang ${ }^{1}$ \\ (1. State Key Laboratory of Geomechanics and Geotechnical Engineering, Institute of Rock and Soil Mechanics, \\ Chinese Academy of Sciences, Hubei Wuhan 430071, China; \\ 2. School of Electronic Information Wuhan University, Hubei Wuhan 430072, China)
}

\begin{abstract}
As a breakthrough in borehole imaging technology, digital panoramic borehole camera technology has been widely employed. The high-resolution panoramic borehole images can accurately reproduce the geometric features of structural planes. However, the detection of these features is usually done manually, which is both time-consuming and introduces human errors. To solve this problem, this paper presents a method for the automatic recognition and parameter extraction of borehole geometric features of camera images. In this method, the image's gray and gradient level, and also their projection on the depth axis are used to identify the locations of structural planes. Afterwards, iterative matching is employed by using a template of sinusoidal function to search for structural planes in the identified image blocks. Finally, optimal sine curves are selected as the feature curves of structural planes, and their related parameters are converted into structural plane parameters required for engineering, such as their positions, dip directions, dip angles and fracture widths. The method can automatically identify all of structural planes throughout the whole borehole camera image in a continuous and rapid manner, and obtain the corresponding structural parameters. It has proven highly reliable, accurate and efficient.
\end{abstract}

Keywords: borehole image; structural plane; regional partitioning; automatic recognition; sine curve

* Corresponding author: Chuanying Wang

Corresponding E-mail: chywang@whrsm.ac.cn

Corresponding Tel: +8613517105001 


\section{Introduction}

Borehole imaging technology has been widely employed in geotechnical, geological, civil engineering, mining, hydropower, oil and geological disaster prevention projects (Cunningham et al., 2004; Genter et al., 1997; Williams and Johnson, 2004; Yang et al., 2015; Zohreh et al., 2014). Based on optical imaging technology, our self-developed digital panoramic borehole camera system is capable of simultaneously observing 360 degrees of a drilling hole, and can generate a large number of high-precision borehole images (WANG et al., 2010; Han et al.). These borehole images can accurately reflect the morphology and geometric parameters of structural planes in the borehole walls (Han et al., 2013; Wang et al., 2002). In engineering practice, due to lack of efficient methods, the post processing of borehole images tend to be tedious and time-consuming work (Liu et al., 2015; Lofi et al., 2012; Schepers et al., 2001). Therefore, we have a practical need to develop the automatic recognition and parameter extraction of structural planes in the borehole camera image.

At present, the extraction of morphological features of borehole images and the extraction of geometric parameters are basically reliant on manual work and human recognition. Structural plane recognition generally involves either using the computer to fit a template of sine curve based on three human-assigned control points, or other combinations of human analysis and computer recognition (Malone et al., 2013). These automatic or semi-automatic operations tend to introduce numerous human factors, which makes them both inefficient and dependent on human operators. Image processing methods including image space transformation, filtering and enhancement, image segmentation, Hough transformation and parameter extraction, when coupled with pattern recognition, can obtain one definite set of structural plane parameters. These methods have improved the recognition process of structural planes with the guidance of some human-determined parameters and the help of human interventions (Assous et al., 2014; Hurich and Deemer, 2013; Klee et al., 2011; Yan et al., 2014). For some boreholes that are several kilometers deep, the highly variable geological structures and large amount of data makes the manual processing work extremely time-consuming (Jia et al., 2012; Zheng et al., 2015). The key to achieving continuous automatic recognition is to find feature parameters that can effectively aid the identification of structural planes (Özkaya, 2002; Yang and Chen, 2009). 
In this paper, we propose a new method of automatic recognition and parameter extraction for structural planes in borehole images. It takes the gray and gradient level, and their vertical distribution data to extract the maximum/minimum gray level values and the maximum gradient value at each pixel row in the image. These values are used to generate new feature signals which can be used for the block-partitioning and feature extraction of the structural planes. The method will also automatically adapt to color variations in the images (Chen et al., 2013; Yang et al., 2015). As structural planes have similar appearances to sine curves in the images, a template of sinusoidal function is used for iterative matching, resulting in optimal sine curves with parameters that can best describe the structural planes.

\section{Feature analysis of structural planes}

Borehole images are indirect reflections of the boreholes walls, and the image features are determined by the geological structure of the walls. Hence they can be divided into two parts, the rock bodies and the structural planes. Due to the inherently different properties of rocks, their mineral particles display different colors in the images. Dark-hued rocks or minerals reflect less light, and leave dimmer images. Light-hued rocks or minerals reflect more light, and leave brighter images (Prensky, 1999; Zheng et al., 2014). Structural planes are generally shown as black ribbons or curves, and they are highly prominent in the images. During the travel of the probe of borehole camera system, its micro CCD camera receives light reflected by the borehole walls. The different reflective properties of rocks or other fillings give structural planes differing brightness in the image. If the structural plane is cemented by quartz feldspar into chunky walls, the incident light can be well reflected, and the resulting images will be bright and easy to read. If the structure is filled with mud and sand, much of the incident light will be reflected diffusely, and the images will be darker. If the structural plane is open without filling or cementation, the incident light cannot be reflected back to the CCD, and the plane will show as a dark block in the image. The "structural planes" refer to ribbon-shaped structural planes that resemble sine curves in borehole images, which is captured by the digital panoramic borehole imaging system developed by the Institute of Rock and Soil Mechanics, Chinese Academy of Sciences.

Different structural planes have different morphological features. In borehole images, these are primarily represented by the hues, shapes and widths of the 
sinusoidal ribbons, and the distribution of patterns and textures surrounding the ribbons. The intersections of structural planes and the borehole are approximately ellipses in the three-dimensional space. When expanded onto a two-dimensional image, they become sine curves (Glossop et al., 1999; Thapa et al., 1997). Hence a standard structural plane is depicted in borehole images as a horizontally extending standard sine curve, while non-standard planes can be approximated as sine curves within a reasonable range, as shown in Fig. 1(a). Therefore, all structural planes can be expressed by using a template of sinusoidal function $y(x)$, as shown in Equation (1). In addition, a structural plane can be described using four parameters: the scanline pixel depth or location of the plane Pos, the dip direction $\alpha$ (the angle between the clockwise direction of the structural plane and the true north), the dip angle $\beta$ (the angle between the structural plane and the horizontal plane), and the facture width $d$. These planes' parameters correspond to the sine curve's coordinate position $i$, phase $\theta$, amplitude $A$, and deviation range $\Delta$. Their conversion relations are shown in Equations (2) to (5), and their correspondences are shown in Fig. 1(b).

$$
\begin{gathered}
y(x)=y_{0}-A \sin (w x+\theta) \\
P o s=\mathrm{k}\left(i+l_{0}\right) \\
\alpha=\left\{\begin{array}{l}
\theta+270^{\circ}, 0^{\circ} \leq \theta<90^{\circ} \\
\theta-90^{\circ}, 90^{\circ} \leq \theta<360^{\circ}
\end{array}\right. \\
A=\frac{D}{2 \mathrm{k}} \tan \beta \quad\left(0 \leq \beta<90^{\circ}\right) \\
d=\frac{\mathrm{k} \Delta}{\cos \beta}=\frac{\mathrm{k}\left(i_{\max }-i_{\min }\right)}{\cos \beta}
\end{gathered}
$$

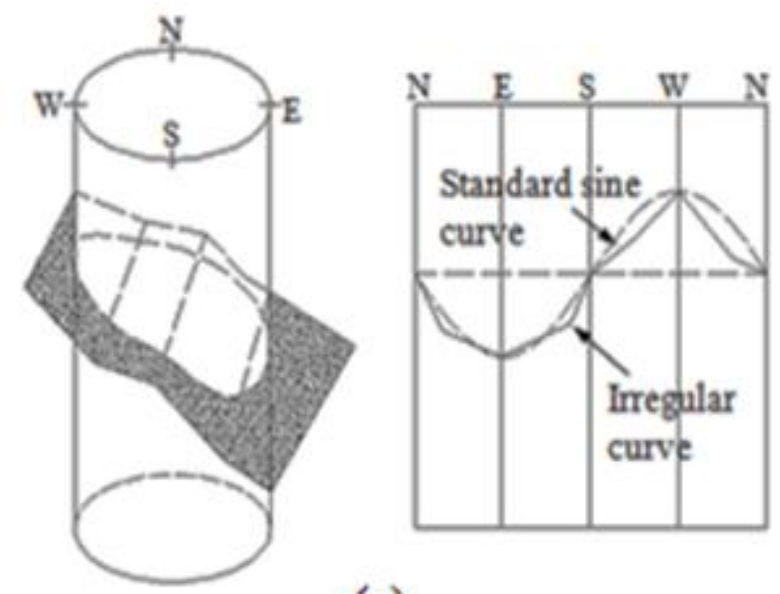

(a) 

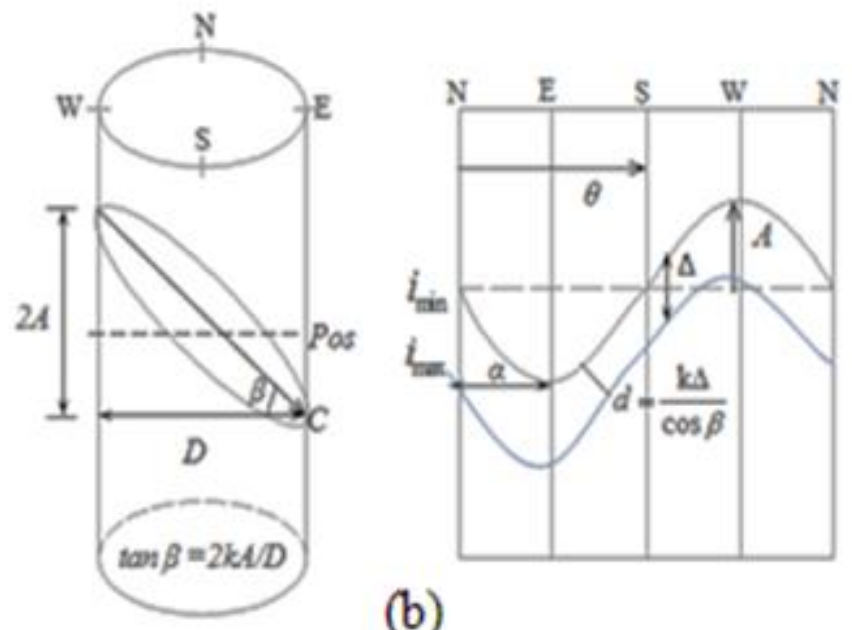

(b)

Fig. 1 The relationship between the structural plane and the sine curve, (a) The sine curve band of structural plane in borehole image, (b) Relations between geometric parameters

In Equations (1) to (5), $y_{0}$ is the initial position of the sinusoidal function, corresponding to the scanline row coordinate $i$ of the function's center in the image; $w$ is the angular frequency of the sinusoidal function, which is the ratio of $2 \pi$ to the number of pixels in each scanline row $N ; l_{0}$ is the initial scanline position from which the curve is cut out from the borehole image; and $\mathrm{k}$ is the resolution of the borehole image in along the depth, i.e. the interval between scanlines, which is a parameter of the drilling equipment. $D$ is the diameter of the borehole and it is $91 \mathrm{~mm}$ in this paper. For the central lines of a structural plane's sine curve band, the maximum and minimum possible scanline coordinates are called as $i_{\max }$ and $i_{\min }$, respectively.

\section{The automatic identification and parameter extraction method}

Based on the feature analysis of structural planes in the image, it has been established that the structural planes are represented as curved bands similar to sine curves (as shown in Fig. 1). Therefore, we can use a template of sinusoidal function to iterate through the feature points on the structural planes and their surrounding areas, to determine whether the sine curve generated by the sinusoidal function is consistent with the structural plane being fitted by counting the number of the feature points. In order to aid the parameter selection and the determination of each structural plane's target block, a cluster-based approach is used to search for new feature variables that describe the structural planes. Using these approaches, the automatic recognition and extraction of geometric parameters can be achieved by using following steps as show in Fig. 2. 


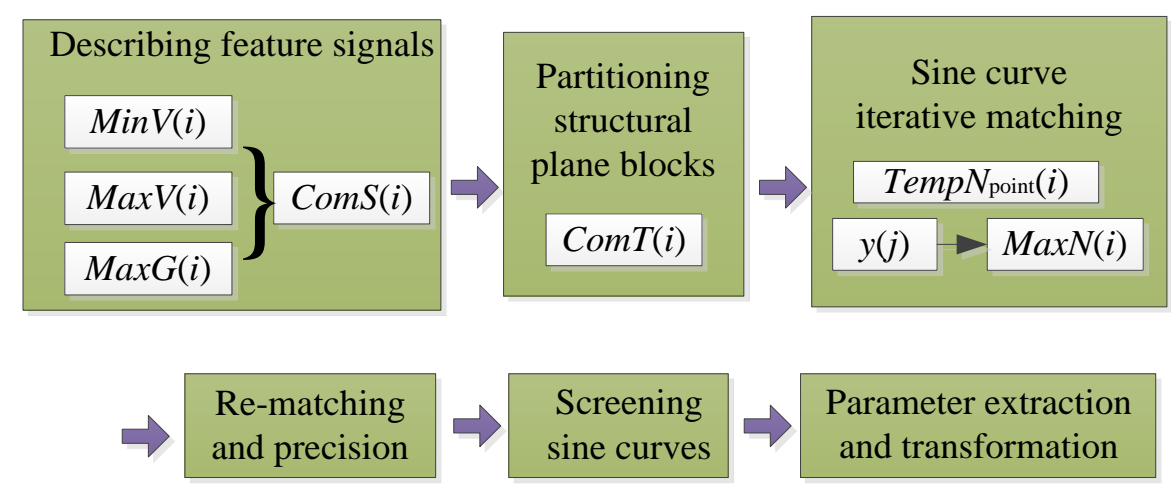

Fig. 2 The steps of automatic recognition and parameter extraction method for structural planes in the borehole camera image

\subsection{Describing feature signals}

The structural planes in borehole images are represented by relatively obvious dark or bright bands, which are denoted our target blocks. The texture of rocks would inevitably create features and spots that are uneven in brightness and sizes in the images, which may affect the recognition of target blocks. However, the structural plane bands in target blocks tend to the darkest or brightest sprites. They are relatively concentrated and continuously distributed along the horizontal axis of the image. By creating a projection of the image onto the vertical depth axis, these feature points can be concentrated into small, comparatively independent blocks. In most cases, planes are separated by large intervals that do not overlap with each other. Therefore, the bands can be easily distinguished from the rock texture noise and the overlapping planes can be considered to be one segment in this step.

In order to prevent the feature bands from being lost among the noise, the minimum and maximum gray levels of each pixel row are first used to describe the structure. The upper left corner pixel in the image is denoted as the origin point of the coordinates. The image is denoted as $f(x, y)$, with $f(i, j)$ being the gray value of the pixel at coordinates $(i, j)$. In the borehole image, the minimum gray value of each row is selected as the minimum eigenvalue of the row using Equation (6). The maximum gray value of each row is selected as the maximum eigenvalue of the row using Equation (7). Here $\operatorname{Min} V(i)$ is the minimum pixel value of the $i^{\text {th }}$ line, $\operatorname{Max} V(i)$ is the maximum pixel value of the $i^{\text {th }}$ line, and $N$ is the width of the image, which is a constant. In the example used in this paper, $N$ is the constant 1024. 


$$
\begin{array}{cc}
\operatorname{Min} V(i)=\min \{f(i, 0), f(i, 1), f(i, 2), \cdots, f(i, j), \cdots, f(i, N-1)\} & (i \in \mathbf{N}) \\
\operatorname{Max} V(i)=\max \{f(i, 0), f(i, 1), f(i, 2), \cdots, f(i, j), \cdots, f(i, N-1)\} & (i \in \mathbf{N})
\end{array}
$$

The gradients of gray levels are used to further describe the gray level variations between the structural plane band and surrounding texture. In the 3 by 3 surrounding area of coordinates $(i, j)$, the horizontal (x axis) gradient is described by Equation (8), and the vertical (y axis) gradient is described by the Equation (9). For the purpose of large-scale processing, the sum of the absolute values of gradients on the $\mathrm{x}$ and $\mathrm{y}$ axes is used as a pixel's gradient value, as shown in Equation (10), where $G_{x}(i, j)$ is the gradient value of the x axis, $G_{y}(i, j)$ is the gradient value of the y axis, and $G(i, j)$ is the gradient value of the pixel $(i, j)$.

$$
\begin{gathered}
G_{x}(i, j)=\frac{\partial f}{\partial x}=\sum_{k=-1}^{1}[f(i+k, j+1)-f(i+k, j-1)] \quad(i, j, k \in \mathbf{N}) \\
G_{y}(i, j)=\frac{\partial f}{\partial y}=\sum_{k=-1}^{1}[f(i+1, j+k)-f(i-1, j+k)] \quad(i, j, k \in \mathbf{N}) \\
G(i, j)=\nabla f=\left[\left(\frac{\partial f}{\partial x}\right)^{2}+\left(\frac{\partial f}{\partial y}\right)^{2}\right]^{1 / 2} \approx\left|G_{x}(i, j)\right|+\left|G_{y}(i, j)\right|
\end{gathered}
$$

The maximum gradient value of each row of pixels can be expressed as the Equation (11), where $\operatorname{Max} G(i)$ is the maximum gradient value for the $i^{\text {th }}$ row.

$$
\operatorname{Max} G(i)=\max \{G(i, 0), G(i, 1), G(i, 2), \cdots, G(i, j), \cdots, G(i, N-1)\} \quad(i \in \mathbf{N})
$$

The minimum and maximum gray values of each row represent the two brightness extremes of the row, which are generally located within the structural planes' sine curve bands, and relatively concentrated when the image is projected onto the vertical axis. In order to further highlight the extremes, we use the difference between $\operatorname{Max} V(i)$ and $\operatorname{Min} V(i)$ of each row as its reference value, and add it to the row's maximum gradient $\operatorname{Max} G(i)$ or its multiplication to form the row's composite signal eigenvalue $\operatorname{ComS}(i)$, as shown in Equation (12).

$$
\operatorname{ComS}(i)=\operatorname{Max} V(i)-\operatorname{Min} V(i)+\lambda \cdot \operatorname{Max} G(i) \quad(\lambda \geq 1, i \in \mathbf{N})
$$

Where: $\operatorname{ComS}(i)$ is the eigenvalue of row $i$, and $\lambda$ is set to 1 according the condition of real borehole images in this paper. This composite signal $\operatorname{ComS}(i)$ is used to represent the distribution of structural planes along the borehole depth. It should be noted that the signals $\operatorname{MaxV}(i), \operatorname{MinV}(i), \operatorname{Max} G(i)$ and $\operatorname{Com} S(i)$ should each undergo filtering and denoising during practice application. One example of the MaxV(i), 
$\operatorname{MinV}(i), \operatorname{Max} G(i)$ and $\operatorname{ComS}(i)$ in practice is shown in Fig. 3. The upper half of Fig.3 is one part of the actual borehole image. The bottom half of Fig. 3 is the described $\operatorname{MaxV}(i), \operatorname{MinV}(i), \operatorname{Max} G(i)$ and $\operatorname{Com} S(i)$ signals. We can see that the maximum gradient value signal $\operatorname{Max} G(i)$ can represent the distribution of structural planes, but the composite signal $\operatorname{Com} S(i)$ is a more effective representation.

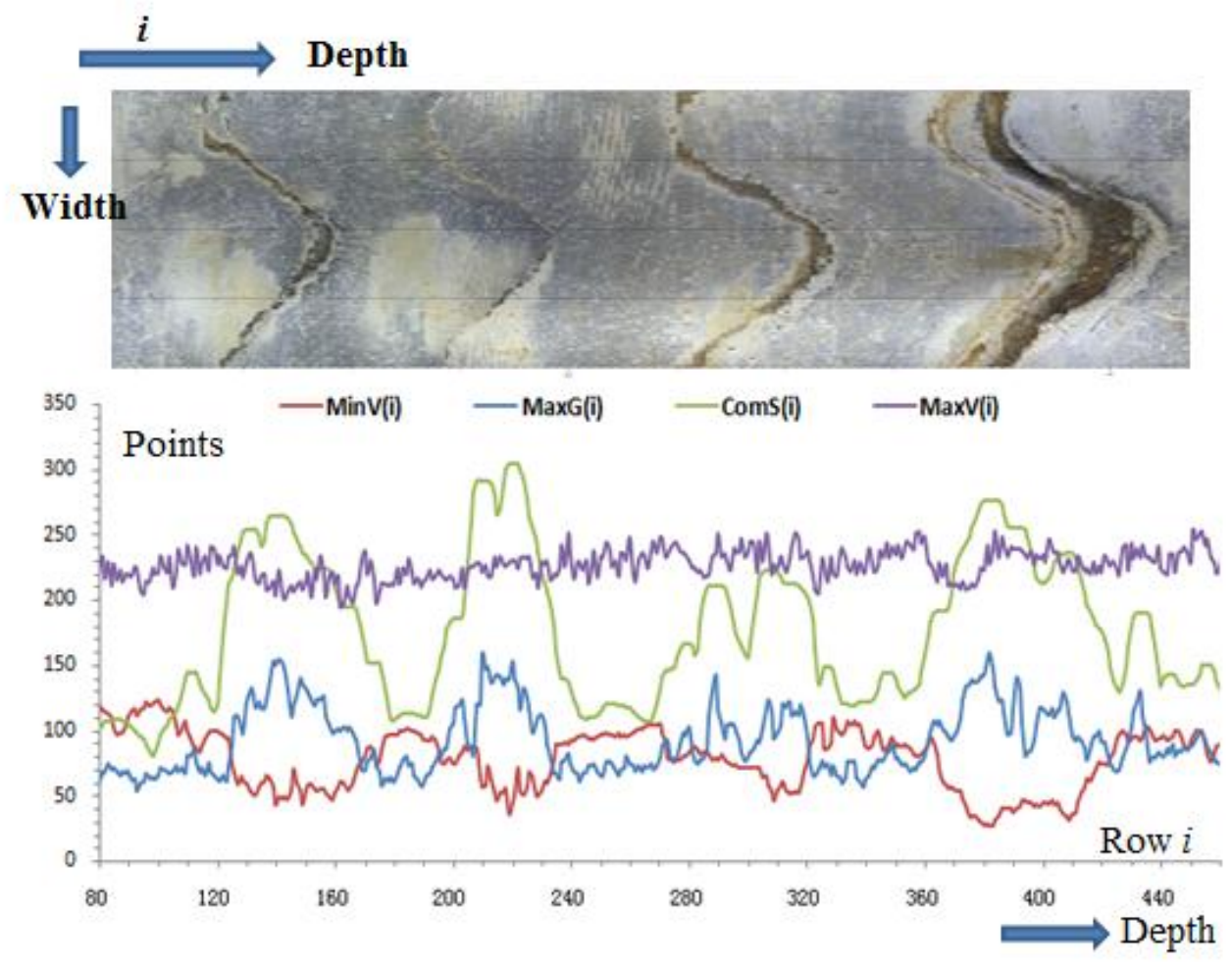

Fig. 3 Comparison between the obtained feature signals and the real borehole image

\subsection{Partitioning structural plane blocks}

Figure 3 has shown that the composite signal $\operatorname{Com} S(i)$ is an effective expression of the structural planes' distribution and general locations of their centers. It is then necessary to perform thresholding on the signal to obtain precise locations of the structural planes. Since the rock textures vary greatly along the several kilometers long borehole, the background brightness of the images also varies. The threshold values of the process must automatically adapt to such changes in the background. For the global threshold of the composite signal, we use the mean of the whole region to be analyzed, as shown in Equation (13). We also use the mean of the rows 10 100 as the local threshold, as shown in Equation (14). For this example, the value of 50 is used according to the condition of real borehole images. 


$$
\begin{gathered}
\overline{\operatorname{Com} T_{\text {global }}}=\frac{1}{M} \sum_{i=0}^{M-1} \operatorname{ComS}(i) \\
\overline{\operatorname{Com}_{\text {local }}}=\frac{1}{2 w} \sum_{k=i-w}^{i+w} \operatorname{ComS}(k) \quad(w \in[10,100])
\end{gathered}
$$

Where: $M$ is the size of the composite signal, or the number of rows in the region to be analyzed; $\overline{\operatorname{Com} T_{\text {global }}}$ is the global threshold; $w$ is the vertical offset, for which 20 is generally sufficient; and $\overline{\operatorname{Com} T_{\text {local }}}$ is the local threshold. Equation (15) shows the thresholding of the composite signal, where $\operatorname{ComT}(i)$ signal is the resultant block signal of the whole borehole image.

$$
\operatorname{Com} T(i)=\left\{\begin{array}{ll}
1, & \operatorname{Com} S(i) \geq\left(\overline{\operatorname{Com} T_{\text {global }}}+\overline{\operatorname{Com} T_{\text {local }}}\right) / 2 \\
0, & \operatorname{ComS}(i)<\left(\overline{\operatorname{Com} T_{\text {global }}}+\overline{\operatorname{Com} T_{\text {local }}}\right) / 2
\end{array} \quad(i \in \mathbf{N})\right.
$$

Using the block signal $\operatorname{ComT}(i)$, the positioning and analysis of sine curves can be easily achieved. When $\operatorname{ComT}(i)$ is not zero, a segment on the vertical axis is considered to contain a structural plane. In addition, smaller blocks can be merged into neighboring large blocks. Based on the composite signal, the block signal has been obtained that allow for partitioning of each structural plane's target block, and positioning of each structural plane's center. The size of a target block indicates the maximum dip angle of the plane, and the coordinates of a plane's center and extreme eigenvalues correspond to the extremes of its sine curve. This allows obtaining a general range for a structural plane's dip direction. These results can be used as the basis for further analysis of the feature sine curves, and simplifies the matching process.

\subsection{Sine curve iterative matching}

A sinusoidal function is used as the template for fitting the structural planes. For each row in a target block, the dip direction $\alpha\left(0^{\circ}-360^{\circ}\right)$ and dip angle $\beta\left(0^{\circ}-90^{\circ}\right)$ are changed using step size $\operatorname{Step}_{1}\left(\operatorname{Step}_{1} \geq 1^{\circ}\right)$, which is set to $1^{\circ}$ in this example. The values are converted using Equations (3) and (4) into the corresponding phase $\theta$ and amplitude $A(A=D /(2 \mathrm{k}) \cdot \tan \beta)$ to be substituted into the sinusoidal function. The gray values of the pixels passed by or surrounding the function curve are computed, and the number of pixels that meet the threshold are counted. Afterwards, the sine curve with the greatest number of pixels that meet the threshold is used as the 
potential sine curve for the row, which is then converted into the corresponding phase and amplitude values, to be used in the template function for fitting with feature points in the borehole image. Finally, one or multiple optimal sine curves in a target block are selected as its structural planes, with one structural plane corresponding to one sine curve.

Using the block signal, we can use the block width $W$ to determine the maximum dip angle $\beta$ of a structural plane, thus defining the range of the amplitude $A$ in the template of sinusoidal function. Further, the center of the structural plane and the $y$-coordinate of the eigenvalue's extreme can be used to arrive at an estimate for the position of the curve's valley value, which gives us the rough range of the dip direction $\alpha$, that is, the approximate range of phase angle $\theta$ of the template of sinusoidal function. This transforms the template function from Equation (1) into Equation (16). This step narrows down the ranges of dip direction and dip angle, and reduces the calculation needed.

$$
y(j)=i-\frac{D}{2 \mathrm{k}} \tan \beta \cdot \sin \left(j \cdot \frac{2 \pi}{N}+\theta\right), \quad j \in[0, N-1], \theta \in(0,2 \pi), \beta \in\left[0, \frac{\mathrm{k} W}{D}\right)
$$

Where: $D$ is the diameter of the borehole, $\alpha$ is the dip direction of the structural plane, and $\beta$ is the dip angle. With the width $W$ of each block and the diameter $D$, the range of dip angle $\beta$ is from 0 to $2 \mathrm{k} W / D$.

When the template of sinusoidal function is employed to match the structural plane, in order to judge whether the pixels passed by or surrounding the template function belongs to the structural plane, a segmentation threshold is employed to distinguish it. This threshold uses the mean of gray values of the block, obtained by Equation (17), where: $m$ is the number of rows in the block, $n$ is the number of columns, $i_{0}$ is the starting row, $i$ is the starting column, and $T_{\text {gray }}$ is the threshold.

$$
T_{\text {gray }}(i)=\frac{1}{m n} \sum_{i=i_{0}}^{i_{0}+m} \sum_{j=j_{0}}^{j_{0}+n} f(i, y(j))
$$

In practice, in order to better utilize the gray scale features of the image, the gray values can be classified into several grades with different weights, using Equation (13), where $Q_{\text {point }}(i, j)$ is the weighted value of the pixel $(i, j)$.

$$
Q_{\text {point }}(i, y(j))=\left\{\begin{array}{l}
0, f(i, y(j))>T_{\text {gary }} \\
1, T_{\text {gary }} \geq f(i, y(j))>T_{\text {gary }} / 2 \\
2, T_{\text {gary }} / 2 \geq f(i, y(j))>T_{\text {gary }} / 4 \\
4, f(i, y(j)) \leq T_{\text {gary }} / 4
\end{array}\right.
$$


The weighted sum of pixels is tallied for the matching of the template $y(x)$, shown in Equation (19), where $\operatorname{Temp}_{\text {point }}(i)$ is the total weighted sum of pixels for the row $i$ under given dip direction and dip angle values. In this example, $N$ is set to a constant 1024 according the Digital Panoramic Borehole Camera System.

$$
\operatorname{TempN}_{\text {point }}(i, \theta, \beta)=\sum_{j=0}^{N-1} Q_{\text {point }}(i, y(j)), \theta \in\left(\theta_{1}, \theta_{2}\right), \beta \in\left[0, \frac{\mathrm{k} W}{D}\right)
$$

Equation (19) gives us a matching score of each function for a row. The function with the highest score is used as the sine curve of the row, and the parameters of its corresponding structural plane are recorded, such as its dip direction, dip angle, position, and the weighted sum score. The variation of the weighted sums along the rows is denoted as the function $\operatorname{MaxN}(i)$. The function $\operatorname{MaxN}(i)$ records the maximum value of the weighted pixel sum for each row's sine curve, shown in Equation (20) .

$$
\operatorname{MaxN}(i)=\max \left\{\operatorname{TempN}_{\text {point }}(i, \theta, \beta)\right\}
$$

The analysis of one selected segment sine curve iterative matching data is shown below in Table 1.

\begin{tabular}{|c|c|c|c|c|c|c|c|c|c|}
\hline No. & $i$ & $\theta /^{\circ}$ & $\beta 1^{\circ}$ & Points & No. & $i$ & $\theta 1^{\circ}$ & $\beta /^{\circ}$ & Points \\
\hline I & I & I & I & I & 8 & 150 & 90 & 48 & 775 \\
\hline 8 & 130 & 66 & 55 & 273 & 9 & 151 & 87 & 46 & 768 \\
\hline 9 & 131 & 153 & 54 & 293 & 30 & 152 & 81 & 45 & 753 \\
\hline 10 & 132 & 145 & 56 & 308 & 31 & 153 & 74 & 47 & 738 \\
\hline 11 & 133 & 142 & 55 & 335 & 32 & 154 & 70 & 45 & 647 \\
\hline 12 & 134 & 139 & 53 & 354 & 33 & 155 & 73 & 46 & 615 \\
\hline 13 & 135 & 79 & 52 & 405 & 34 & 156 & 66 & 44 & 613 \\
\hline 14 & 136 & 83 & 56 & 419 & 35 & 157 & 60 & 44 & 600 \\
\hline 15 & 137 & 85 & 54 & 435 & 36 & 158 & 53 & 43 & 545 \\
\hline 16 & 138 & 94 & 53 & 475 & 37 & 159 & 49 & 41 & 538 \\
\hline 17 & 139 & 95 & 52 & 523 & 38 & 160 & 44 & 42 & 533 \\
\hline 18 & 140 & 100 & 54 & 613 & 39 & 161 & 39 & 43 & 517 \\
\hline 19 & 141 & 101 & 52 & 738 & 40 & 162 & 34 & 44 & 506 \\
\hline
\end{tabular}

Table 1 Parameters of the structural plane sine curves (from a selected segment) 


\begin{tabular}{llllllllll}
20 & 142 & 103 & 50 & 835 & 41 & 163 & 28 & 45 & 487 \\
21 & 143 & 103 & 49 & 786 & 42 & 164 & 24 & 44 & 477 \\
22 & 144 & 108 & 49 & 870 & 43 & 165 & 20 & 45 & 461 \\
23 & 145 & 108 & 48 & 1026 & 44 & 166 & 14 & 44 & 449 \\
24 & 146 & 109 & 47 & 961 & 45 & 167 & 11 & 45 & 437 \\
25 & 147 & 103 & 45 & 933 & 46 & 168 & 7 & 45 & 438 \\
26 & 148 & 117 & 46 & 859 & 47 & 198 & 173 & 47 & 85 \\
27 & 149 & 95 & 48 & 835 & $/$ & $/$ & $/$ & $/$ & $/$ \\
\cline { 7 - 9 }
\end{tabular}

\subsection{Screening the sine curves}

From Table 1, it can be seen that generally a sine curve with the most matching feature points have been found for each row, and one of them is the sine curve that best describes the given structural plane. Obviously, the sine curve with the most matching feature points is the one that best describes the features of a plane. It is also possible for a block to contain multiple fissures or structural planes, thus structural planes may also be found in places with the most frequent occurrence of matching points and the greatest variations. Therefore, depending on the dip and positions of the sine curves, we can select curves that correspond to maximum or extreme values in $\operatorname{MaxN}(i)$ as the representative sine curves. As shown in Fig. 4, we can see that 6 blocks are found in the $\operatorname{MaxN}(i)$ signal.

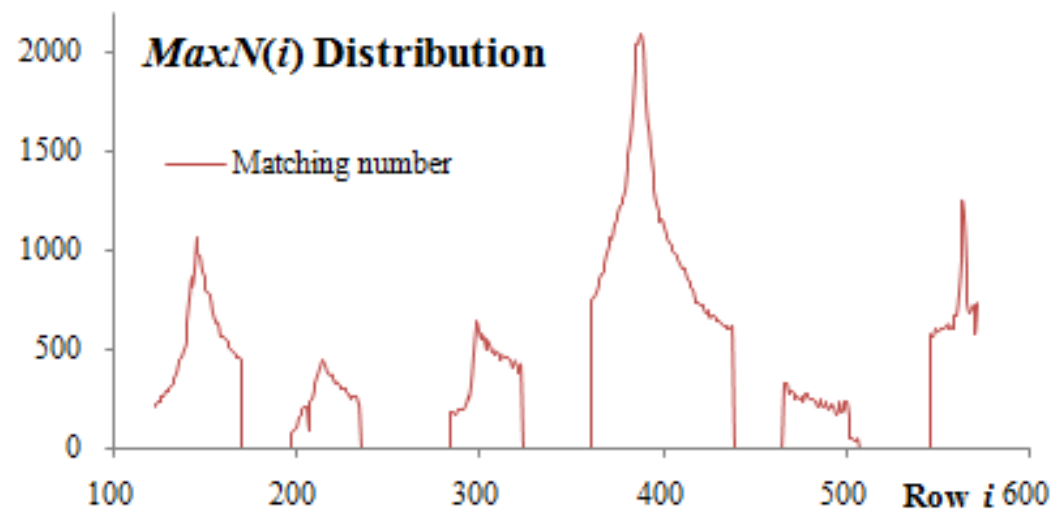

Fig. 4 The distribution of the matching signal $\operatorname{MaxN}(i)$

One block usually possesses one extreme point as shown in Fig. 4 and sine curves 
corresponding to the extremes are used to represent the structural planes. The results are shown in Table 2. The first row in the table is the result of screening the data from Table 1, and the second to the sixth rows are from omitted data of Table 1.

Table 2 Parameters from the of recognition of structural planes

\begin{tabular}{ccccc}
\hline No. & Row $i$ & Dip direction $\theta /^{\circ}$ & Dip angle $\beta /^{\circ}$ & Matching Points \\
\hline 1 & 145 & 108 & 48 & 1026 \\
2 & 213 & 111 & 49 & 437 \\
3 & 298 & 119 & 51 & 636 \\
4 & 387 & 118 & 56 & 2083 \\
5 & 466 & 120 & 43 & 324 \\
6 & 565 & 105 & 37 & 1315 \\
\hline
\end{tabular}

\subsection{Re-matching and precision}

In the above process, if the matching step size Step $L_{1}$ is larger, the number of iterations will be smaller. In order to reduce the time used in the matching process of Section 2.3, a relatively large value has been used for StepL $L_{1}$, which limits the precision of the results above. To achieve higher accuracy, it is necessary to perform another round of matching in a narrower range. Constant values are added or subtracted to the obtained positions, dip directions and dip angles of structural planes to create the new upper and lower limits. For example, the fluctuation range of a sine curve's center is $(i-\mathrm{a}, i+\mathrm{a})$, the range of the dip direction is $(\alpha-\mathrm{b}, \alpha+\mathrm{b})$, and the range of the dip angle is $(\beta-\mathrm{c}, \beta+\mathrm{c})$, where $\mathrm{a}, \mathrm{b}$ and $\mathrm{c}$ are constants and can be changed along the current structural plane blocks. We set initial value of a as 10 according to average value of structural plane's gap width. The constants $b$ and $c$ are set as 2 and 3 respectively according to a bigger value than $S t e p L_{1}$. We then use the step size $S t e p L_{2}$ $\left(\right.$ StepL $L_{1}>$ Step $\left._{2}>0\right)$ to rematch the function, obtain the weighted sum of pixels, and arrive at a more optimal sine curve.

$S t e p L_{2}$ can be based on the actual precision we need. For example, we can set Step $L_{2}=0.1$, and iterate within the ranges of $(i-\mathrm{a}, i+\mathrm{a}),(\alpha-\mathrm{b}, \alpha+\mathrm{b}),(\beta-\mathrm{c}, \beta+\mathrm{c})$ by repeating the above steps. In this example, the re-matching is performed, with the final results are shown in Table 3. The final curves are shown in Fig. 5, where the red 
curves are the matched sine curves of the structural planes in the borehole image.

Table 3 Results of re-matching and screening

\begin{tabular}{ccccc}
\hline No. & Row $i$ & Dip direction $\theta /^{\circ}$ & Dip angle $\beta /^{\circ}$ & Matching Points \\
\hline 1 & 145 & 108.7 & 47.8 & 1064 \\
2 & 213 & 111.2 & 49.3 & 471 \\
3 & 298 & 119.4 & 51.7 & 673 \\
4 & 387 & 117.8 & 56.4 & 2194 \\
5 & 466 & 120.5 & 43.2 & 352 \\
6 & 565 & 104.6 & 36.8 & 1347 \\
\hline
\end{tabular}

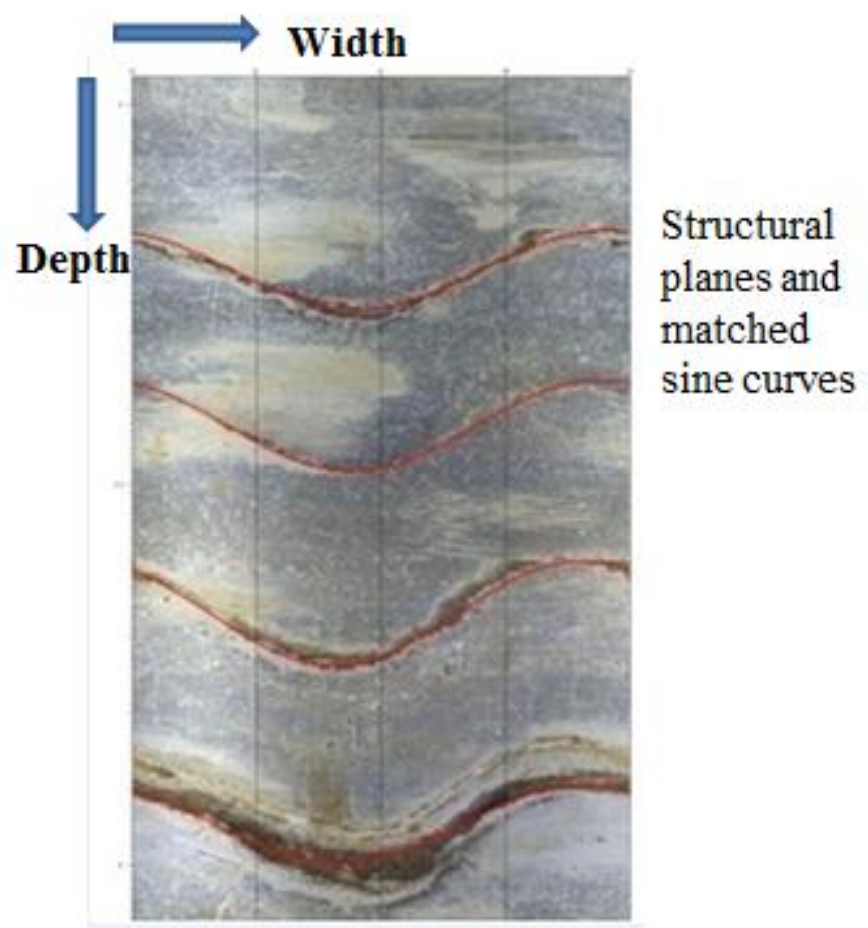

Fig. 5 The matching result between the sine curves and the structural planes

\subsection{Parameter extraction and transformation}

In the above process, the final resultant pixel coordinate is the row coordinate $i$, representing the position of the sine curve, which can be converted into the actual depth of the structural plane Pos using Equation (2). The resultant dip angle is the dip angle $\beta$ of the structural plane. The transformation between the dip angle $\beta$ and the amplitude $A$ of the sine curve is shown in Equation (4). The dip angle $\alpha$ of the 
structural plane is the angle between the side length (amplitude $A$ ) corresponding to the curve's peak and the radius. The dip direction $\alpha$ of the structural plane is the angle between the clockwise direction corresponding to the sine curve's value and the true north, the relationship between which and the curve's phase $\theta$ is shown in Equation (3). The dip direction $\alpha$ of the structural plane can be further converted into the $\operatorname{dip} \varphi$ using Equation (21), which makes it easier to understand during practice application.

$$
\varphi= \begin{cases}\mathrm{NE}: \alpha, & 0^{\circ} \leq \alpha<90^{\circ} \\ \mathrm{SE}: 180^{\circ}-\alpha, & 90^{\circ} \leq \alpha<180^{\circ} \\ \mathrm{SW}: \alpha-180^{\circ}, & 180^{\circ} \leq \alpha<270^{\circ} \\ \mathrm{NW}: 360^{\circ}-\alpha, & 270^{\circ} \leq \alpha<360^{\circ}\end{cases}
$$

The gap width $d$ of the structural plane gap is calculated as follows. For each structural plane, the row coordinate of the intersection between the sine curve and the upper edge is the minimum offset $i_{\min }$, and the row coordinate of the intersection between the sine curve and the lower edge is the minimum offset $i_{\max }$. The gap width is related to the fluctuation range $\Delta$ of the sine curve in the image, where $\Delta=i_{\max }-i_{\min }$. This is calculated using Equation (5). Table 4 shows the widths and other resultant parameters of the structural planes 1 to 6 from Table 3 .

Table 4 Related parameters of automatic recognition for structural planes in the borehole image

\begin{tabular}{ccccccc}
\hline No. & Depth $/ \mathrm{m}$ & Row $i$ & Points & Dip direction $\alpha /^{\circ}$ & Dip angle $\beta /^{\circ}$ & Gap width $d / \mathrm{mm}$ \\
\hline 1 & -3.218 & 145 & 1064 & 161.3 & 47.8 & 9.2 \\
2 & -3.423 & 213 & 471 & 157.5 & 49.3 & 2.7 \\
3 & -3.678 & 298 & 673 & 150.2 & 51.7 & 12.5 \\
4 & -3.979 & 387 & 2194 & 152.6 & 56.4 & 23.8 \\
5 & -4.184 & 466 & 352 & 148.3 & 43.2 & 5.6 \\
6 & -4.476 & 565 & 1347 & 164.7 & 36.8 & 7.4 \\
\hline
\end{tabular}

\section{Result analysis and discussion}

\subsection{Analysis of structural plane partitioning}

In the past, the method of borehole image recognition is generally employed to 
identify and compare the specific regions that are expected to be realized. Such methods are often difficult to meet the requirements of the actual project, which requires a priori knowledge of the staff and human intervention. One advantage of the method in this paper is that it can extract the feature information of the whole borehole image, and extract the structural information, so as to realize the regional division of the structure plane and the collection and processing of information. How to correctly and effectively divide the region of the structure is one of the key issues to improve the accuracy and speed.

In the borehole image, the rock, especially the smooth hole-wall of the rock, is reflected by massive specular reflection, and the image is bright, and the gray value is higher. However, the structure plane of the pores due to the reflection of the back light is less and the performance is dim, the image gray level is generally low, which is the target feature region in borehole image. In order to effectively highlight the low gray target feature region, the method selects a minimum gray value of the pixel points to represent the target feature region, and selects a maximum gray value pixel to represent the image. These maximum and minimum gray gradient pixels are the feature points of borehole image, and constitute the maximum signal $\operatorname{MaxV}(j)$, minimum signal $\operatorname{Min} V(j)$, maximum gradient signal $\operatorname{Max} G(j)$, synthetic signal $\operatorname{Com} S(j)$ and block signal $\operatorname{Com} T(j)$. These signals effectively describe the borehole image features and the distribution of structure plane. Because of the complex diversity of rock and structure, there is a large amount of noise signals in borehole image. To some extent, the noise can also affect the feature signal, so the appropriate signal filtering and smoothing processing can more correctly grasp the main features of the structure plane of the borehole image, so as to realize the high efficiency of the structure plane.

However, in the borehole images, there are some discontinuous structural planes or fractured, ruptured discontinuities, which lead to the partial loss of feature signals. When the borehole image is segmented by vertical projection, there partly are the cross structure planes or the relatively parallel connection of structure planes in the borehole image. Thus, in the synthesis signal $\operatorname{Com} S(j)$ and block signal $\operatorname{Com} T(j)$, there are some isolated small feature regions, as shown in Fig. 6 (a). These relatively small and isolated regions are generally the imaging of small rocks caused by the hole, but it does not rule out that the signal of the structure plane caused by the discontinuous structural planes or fractured, ruptured discontinuities. In order to reduce the loss of 
feature signals from structural plane as much as possible, these small isolated feature regions are necessary to keep, and should not be filtered out as the noise. In this paper, we take the method of "nearest principle", that is, divide the block into the nearest block or the next block, and connect with it as a whole, and thus ensure the integrity of the signal, as shown in Fig. 6 (b).

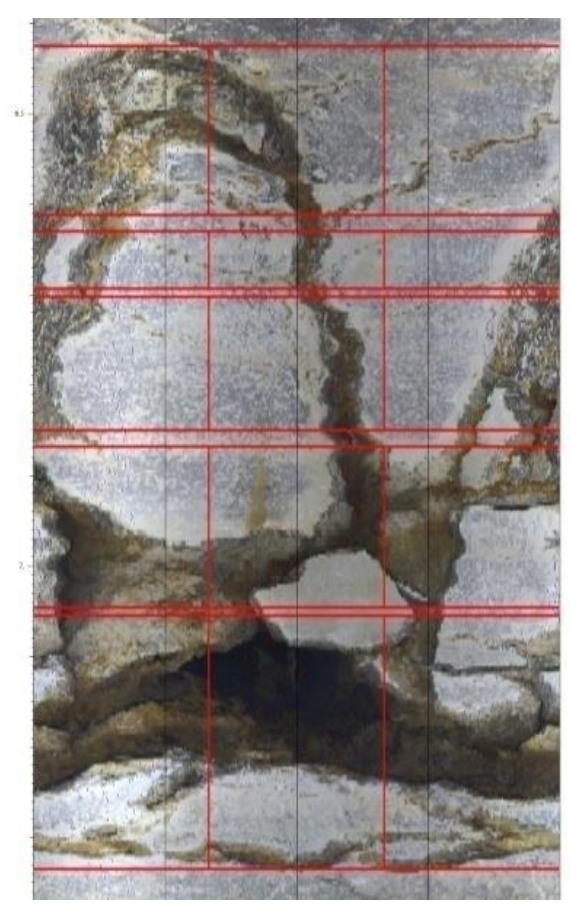

(a)

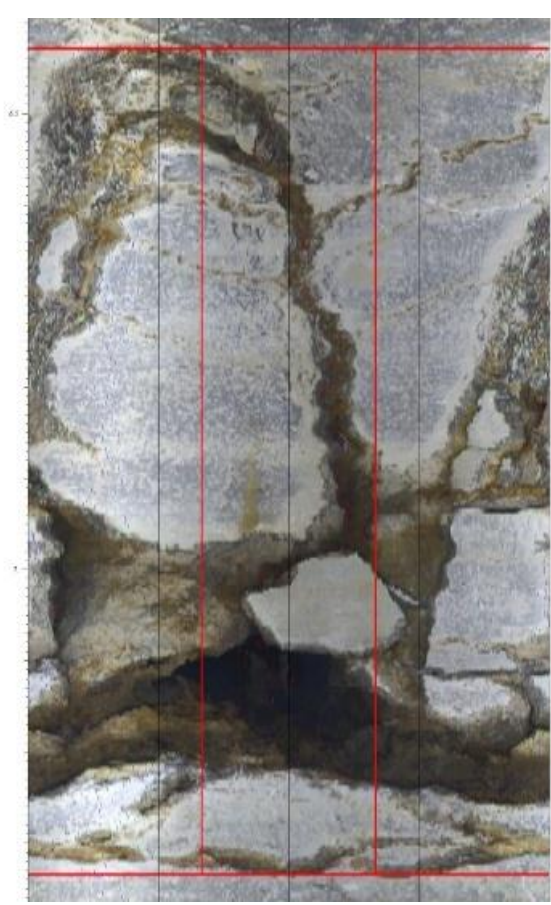

(b)

Fig. 6 Effects of isolated small blocks on the partitioning of structural planes, (a) Before merging, (b) After merging

In addition, in this paper, the mean value of the local mean and that of the global mean is adopted as the matching threshold. It can effectively change the borehole image, and achieve the accurate recognition and parameter extraction of structure plane features. The effective partition and feature information of the structure plane can greatly improve matching efficiency.

\subsection{Selection of sine curves}

After using the template of sinusoidal function to perform iterative matching on the feature points of each structural plane within one block, a sine curve with the highest matching numbers has been selected for each pixel row in a target block. These sine curves need to be further screened to find the sine curve that best describes the structural plane. However, as closely parallel, intersecting or broken planes are often found in real images, more than one plane may exist in a block, and using only 
one curve to represent a block causes the loss of structural planes.

In order to solve the situation that one or more planes may exist in a block, this method selects one or more sine curves corresponding to the maximum or extreme values of the block signal $\operatorname{MaxN}(i)$ to represent one or more structural planes. And then we differentiate the multiple planes by using the different positions, phases and amplitudes of the sine curves (Deltombe and Schepers, 2004). If the maximum values, or nearby maximums of $\operatorname{MaxN}(i)$ correspond to curves with similar phases and amplitudes but significantly different position values, then several parallel structural planes are present. If their phases, amplitudes and positions are all significantly different, then several intersecting planes are present. For example, Fig. 7 (a) shows the results and corresponding $\operatorname{MaxN}(i)$ signals for intersecting planes in a block, and Fig. 7 (b) shows the results and signals for parallel planes. The related curve signals and signals are shown in Figs. $7 \sim 8$. The related data are shown in Table 5.

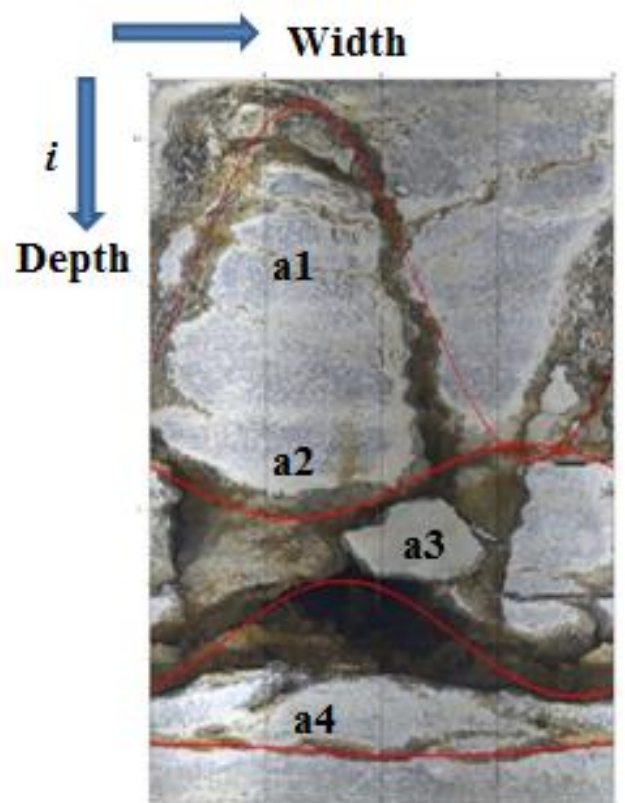

(a)

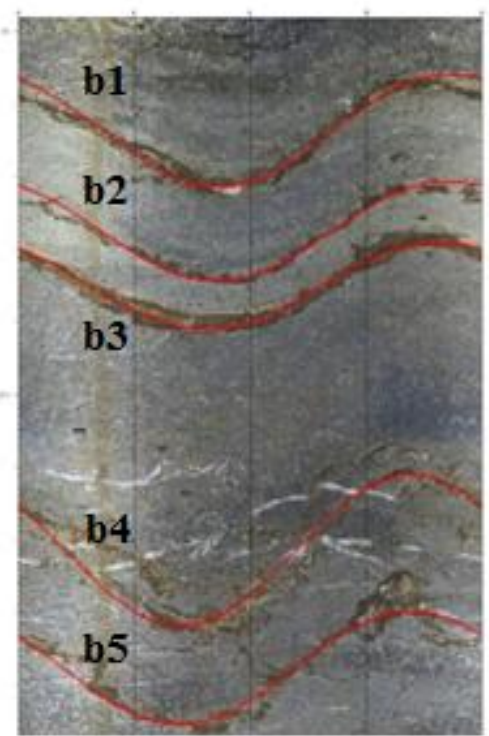

(b)

Fig. 7 Recognition results of structural planes, (a) Irregular, complex, intersecting structural planes, (b) Regular, simple structural planes

In Figs. 7, the exist structural planes of Fig. 7(a) are denoted as a1, a2, a3 and a4, respectively, and the exist structural planes of Fig. 7(b) are denoted as b1, b2, b3, b4 and b5, respectively. The $\operatorname{MaxN}(i)$ and $\operatorname{ComT}(i)$ signals of Fig. 7 (a) is shown as the curves of $\operatorname{MaxN}(i) \_$a and $\operatorname{ComT}(i) \_$a in Fig. 8. The $\operatorname{MaxN}(i)$ and $\operatorname{ComT}(i)$ signals of Fig. 7 (b) is shown as the curves of $\operatorname{MaxN}(i) \_b$ and ComT(i)_b in Fig. 8. 
From Fig. 8, we can see that the curve of $\operatorname{Com} T(i) \_$a signal exists a block, and there are 4 extreme points of curve $\operatorname{Max} N(i)_{-}$a within the block. The 4 extreme points (i. e. the positions of words a1, a2, a3 and a4 in Fig. 8) of curve $\operatorname{MaxN}(i)$ a can correspond to 4 sine curves. The relevant parameters of the 4 sine curves are used to represent the geometry parameters of structural planes in borehole image. The related data are shown as row No. a1, a2, a3 and a4 in Table 5. So as to the curve $\operatorname{MaxN}(i) \_b$ and ComT $(i)$ b signal. What differences are that the ComT $(i)$ b b signals exists two blocks. There are 3 extreme points of curve $\operatorname{MaxN}(i) \_b$ under the first block of ComT $(i) \_$b. And there are 2 extreme points of curve $\operatorname{MaxN}(i) \_b$ under the second block of $\operatorname{ComT}(i) \_$b. These 5 extreme points of curve $\operatorname{MaxN}(i) \_\mathrm{b}$ correspond to 5 sine curves. The relevant parameters of the 5 sine curves are used to represent the geometry parameters of structural planes in borehole image. They are shown as row No. b1, b2, b3, b4 and b5 in Table 5.

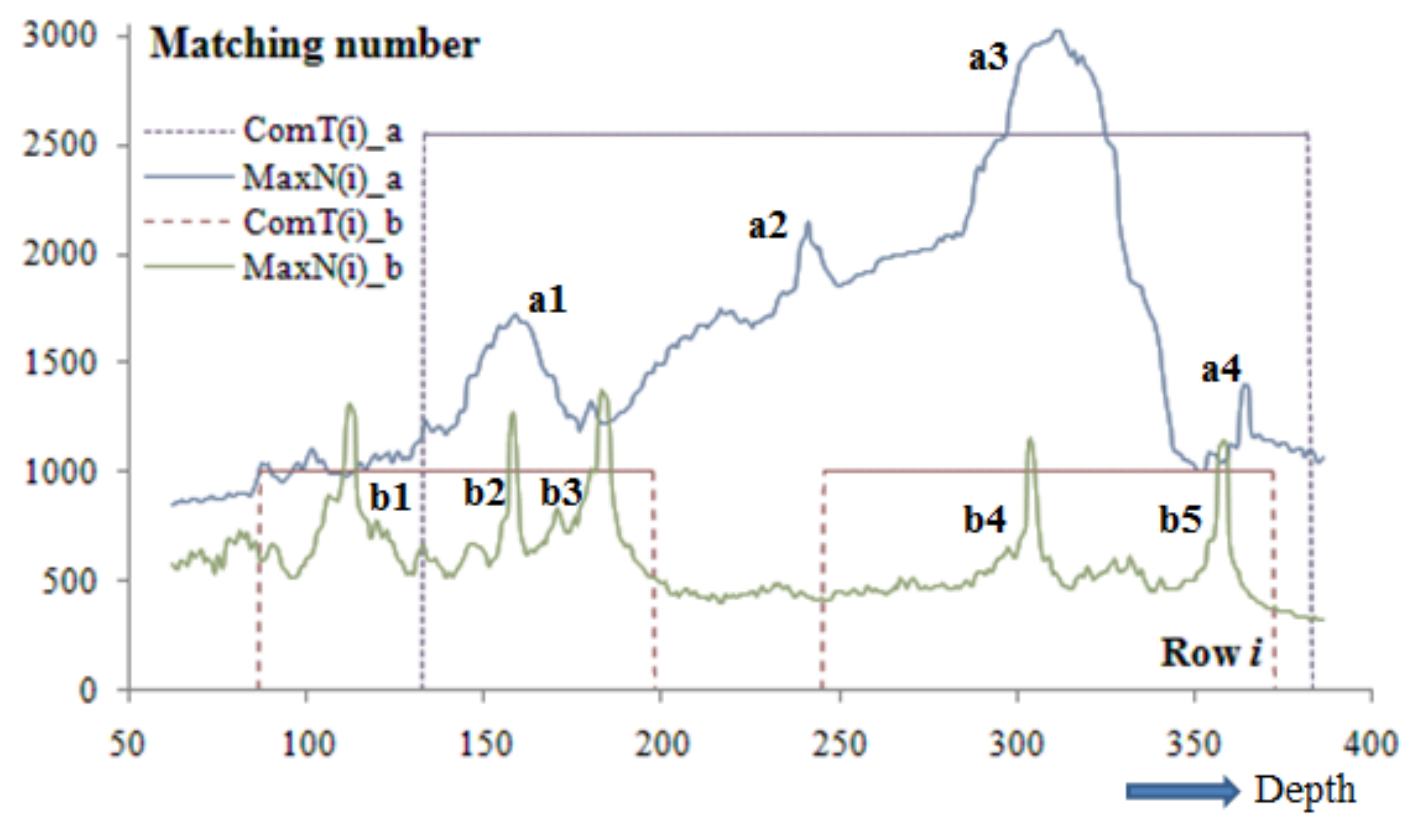

Fig. 8 The distribution of matching number signal $\operatorname{MaxN}(i)$ and block signal $\operatorname{Com} T(i)$ for parallel and intersecting structural planes

Table 5 Parameters of automatically recognized structural planes in Fig. 7

\begin{tabular}{cccccc}
\hline No. & Depth $/ \mathrm{m}$ & Matching point & Dip direction $\alpha /^{\circ}$ & Dip angle $\beta /{ }^{\circ}$ & Gap width $d / \mathrm{mm}$ \\
\hline $\mathbf{a 1}$ & -6.695 & 1721 & 332.4 & 79.2 & 22.6 \\
a2 & -6.955 & 2143 & 157.3 & 47.5 & 20.3 \\
\hline
\end{tabular}




\begin{tabular}{llllll}
\hline $\mathbf{a 3}$ & -7.164 & 3022 & 300.6 & 59.6 & 98.5 \\
$\mathbf{a 4}$ & -7.320 & 1392 & 62.2 & 10.3 & 9.2 \\
$\mathbf{b 1}$ & -32.141 & 1315 & 115.7 & 58.4 & 13.4 \\
$\mathbf{b 2}$ & -32.276 & 1270 & 116.8 & 57.2 & 8.3 \\
$\mathbf{b 3}$ & -32.351 & 1370 & 124.1 & 55.8 & 24.8 \\
$\mathbf{b 4}$ & -32.715 & 1151 & 114.6 & 65.6 & 7.6 \\
$\mathbf{b 5}$ & -32.876 & 1149 & 145.3 & 60.7 & 8.5 \\
\hline
\end{tabular}

Figure 8 and Table 5 show that each $\operatorname{MaxN}(i)$ signal's extreme may correspond to one structural plane. These extreme values can help us select the optimal and most likely structural planes. We can also see in Figs. 7 that the recognized curves in red are generally consistent with the structural planes, and the obtained parameters have met the basic requirements of engineering.

\subsection{Discussion of automatic identification results}

We now know that the method allows for continuous and automatic identification of structural planes in borehole images and their parameters including positions, dip directions, dip angles and gap widths. The results can be compared with traditional manual recognition, where three control points are given by humans on the image, and a standard curve fitting algorithm is applied to the sine. If the curve is consistent with the structural plane, the computer will output its geometric parameters. For the structural planes in Figs. 7, the results of real manual recognition are shown in the left half of Table 6 , and the comparison with related data of Table 5 is shown in the right half Table 6.

Table 6 Comparison between manual and automatic recognition

\begin{tabular}{cccccccccc}
\hline & \multicolumn{4}{c}{ Manual recognition } & \multicolumn{4}{c}{ Deviation compared with Table 5 } \\
No. & Pos $/ \mathrm{m}$ & $\alpha /^{\circ}$ & $\beta / /^{\circ}$ & $d / \mathrm{mm}$ & Pos $/ \mathrm{m}$ & $\alpha /^{\circ}$ & $\beta /^{\circ}$ & $d / \mathrm{mm}$ \\
\hline $\mathbf{a 1}$ & -6.664 & 331.7 & 79.4 & 21.4 & -0.031 & -0.7 & 0.2 & -1.2 \\
$\mathbf{a 2}$ & -6.969 & 157.5 & 46.9 & 18.9 & 0.014 & 0.2 & -0.6 & -1.4 \\
$\mathbf{a 3}$ & -7.171 & 301.1 & 58.7 & 99.3 & 0.007 & 0.5 & -0.9 & 0.8 \\
$\mathbf{a 4}$ & -7.320 & 61.3 & 10.3 & 10.9 & 0.000 & -0.9 & 0.4 & 1.3 \\
\hline
\end{tabular}




\begin{tabular}{lllllllll}
\hline b1 & -32.183 & 116.5 & 57.6 & 14.1 & -0.042 & 0.8 & -0.8 & 1.5 \\
b2 & -32.276 & 117.2 & 56.9 & 8.7 & -0.008 & 0.4 & -0.3 & 0.4 \\
b3 & -32.344 & 124.3 & 55.8 & 24.5 & 0.007 & 0.2 & 0.0 & -0.3 \\
b4 & -32.702 & 114.5 & 65.7 & 9.4 & -0.013 & -0.1 & 0.1 & 1.8 \\
b5 & -32.881 & 144.8 & 61.1 & 9.8 & 0.005 & -0.5 & 0.4 & 1.3 \\
\hline
\end{tabular}

As manual recognition introduces various uncertainties, the averages of multiple repeated recognitions are used as results. In Table 6, the parameters obtained by manual recognition are all average results that were accepted by the working staff as valid and suitable for use as standard parameters. The comparison shows that the method can recognize all structural planes and the results have good consistency with real structural planes. The deviation of depth Pos is less than $0.05 \mathrm{~m}$, the deviation of dip direction $\alpha$ is less than $1^{\circ}$, the deviation of dip angle $\beta$ is less than $1^{\circ}$, and the deviation gap width $d$ is less than $2 \mathrm{~mm}$ in Table 6 . These absolute deviations of the parameters satisfy the requirements of engineering application at present.

In order to show some more automatic recognition results by using this method, we use the automatic recognition and parameter extraction method to deal with the structural planes of borehole image under a clear water borehole with the depth of about $250 \mathrm{~m}$ in Hangzhou Bay, China. Results of automatic recognition are also compared with original manual recognition. We can see that the method of automatic recognition only takes few hours while our manual recognition takes few days. The statistical results of automatic recognition and manual recognition are shown in Table 7.

Table 7 The statistical results of automatic recognition and manual recognition for the same borehole image with depth about $250 \mathrm{~m}$ borehole under clear water

\begin{tabular}{ccccccc}
\hline Working mode & Total & Recognized & Errors & Missed & Deviation & Time \\
\hline Manual recognition & 107 & 107 & 0 & 0 & $0 \%$ & 3 days \\
Automatic recognition & 98 & 87 & 21 & 8 & $4 \%$ & 1.7 hrs. \\
\hline
\end{tabular}

We take all the recognized structural plane sine curves (i.e. 107 sine curves in table 7) as the standard results and the results of automatic recognition by computer are compared with it. The data of table show that this automatic recognition results 
can be accepted in actual application engineering and this method can save plenty of time at least. Furthermore, the method can be used to process an entire borehole image in one session, compared to manual recognition which requires breaking down the image into multiple segments and constant human intervention in the process, which is a breakthrough in efficiency. Without human factors, the results can be more accurate and reliable.

\section{Conclusion}

In this paper, we propose a method for the automatic continuous recognition and parameter extraction of structural planes from the optical borehole image. The method extracts and analyzes the minimum and maximum gray levels and the maximum gradients from each pixel row of the image, and generates two new feature signals, i.e., the composite signals and the block signals, to assist the recognition of structural planes, by partitioning the image into multiple blocks. This is followed by using the template of sinusoidal functions for iterative matching of all possible structural planes in a block, and selecting the sine curves. Through the block partitioning and result screening, the method has effectively realized the automatic recognition of structural planes and extraction of their parameters. The automatically recognized results are consistent with images with images within an accepted recognition rate and related accurate parameters. This method can effective eliminate the need for human intervention, and greatly increase the efficiency of borehole image analysis by saving plenty of time.

The main conclusions are: (1) the block-partitioning of structural planes can be achieved using the projection of the image onto the vertical depth axis; (2) the automatic recognition of structural planes in the image can be achieved by using the template of sinusoidal function to perform iterative matching on structural planes, with a high recognition rate; (3) the division of blocks greatly simplifies the iteration process, and enables the geometric parameter analysis along the whole image; (4) the function matching is a simple and reliable method that can iterate through all possible sine curves; (5) the method can be computationally efficient. It is effective and of practical value for the analysts of the optical borehole images.

\section{Acknowledgments}


This paper was supported by the Strategic Research Program of the Chinese Academy of Sciences (Grant No.XDB10030200) and the National Science Foundation of China (Grant No. 41402278 and No. 41372317), and was also supported by the State Key Development Program for Basic Research of China (Grant No. 2013CB956104).

\section{References:}

Assous, S., Elkington, P., Clark, S. and Whetton, J., 2014. Automated detection of planar geologic features in borehole images. Geophysics, 79(1): D11-D19.

Chen, Z., Qin, Q.Q., Lin, L.Y., Liu, Q. and Zhan, W.F., 2013. DEM Densification Using Perspective Shape From Shading Through Multispectral Imagery. IEEE Geoscience and Remote Sensing Letters, 10(1): 145-149.

Cunningham, K.J., Carlson, J.I. and Hurley, N.F., 2004. New method for quantification of vuggy porosity from digital optical borehole images as applied to the karstic Pleistocene limestone of the Biscayne aquifer, southeastern Florida. Journal of Applied Geophysics, 55(1 - 2): 77 - 90.

Deltombe, J. and Schepers, R., 2004. New developments in real-time processing of full waveform acoustic televiewer data. Journal of Applied Geophysics, 55(1 - 2): 161 - 172.

Genter, A. et al., 1997. Comparative analysis of direct (core) and indirect (borehole imaging tools) collection of fracture data in the Hot Dry Rock Soultz reservoir (France). Journal of Geophysical Research: Solid Earth, 102(B7): 15419--15431.

Glossop, K., Lisboa, P.J.G., Russell, P.C., Siddans, A. and Jones, G.R., 1999. An Implementation of the Hough Transformation for the Identification and Labelling of Fixed Period Sinusoidal Curves. Computer Vision and Image Understanding, 74(1): 96 - 100.

Han, Z., Wang, C., Liu, S. and Zhu, H., 2013. Research on Connectivity of Deep Ore-lodes of Borehole based on Digital Borehole Camera. Disaster Advances, 6(8): 41-46.

Han, Z. Q., Wang, C. Y. and Zhu, H. Y., 2014. Investigation of Deep Joints and Analysis of Lode Extension Direction in Shapinggou Molybdenum Mine. In Applied Mechanics and Materials, 638: 2151-2154).

Hurich, C. and Deemer, S., 2013. Combined surface and borehole seismic imaging in a hard rock terrain: A field test of seismic interferometry. Geophysics, 78(3): B103-B110.

Jia, P., Yang, T.H. and Zhang, C.M., 2012. Discussions on zonal disintegration around tunnel in deep rock mass. Advanced Materials Research, pp. 2285-2289.

Klee, G., Bunger, A., Meyer, G., Rummel, F. and Shen, B., 2011. In Situ Stresses in Borehole Blanche-1/South Australia Derived from Breakouts, Core Discing and Hydraulic Fracturing to $2 \mathrm{~km}$ Depth. Rock Mechanics and Rock Engineering, 44(5): 531-540.

Liu, H.L., Yang, T.H., Xu, T. and Yu, Q.L., 2015. A comparative study of hydraulic fracturing with various boreholes in coal seam. Geosciences Journal, 19(3): 489-502.

Lofi, J. et al., 2012. Geological discontinuities, main flow path and chemical alteration in a marly hill prone to slope instability: Assessment from petrophysical measurements and borehole image analysis. Hydrological Processes, 26(14): 2071--2084. 
Malone, T., Hubbard, B., Merton-Lyn, D., Worthington, P. and Zwiggelaar, R., 2013. Borehole and Ice Feature Annotation Tool (BIFAT): A program for the automatic and manual annotation of glacier borehole images. Computers \& Geosciences, 51: 381 - 389.

Özkaya, S.I., 2002. KINKFOLD - an AutoLISP program for construction of geological cross-sections using borehole image data. Computers \& Geosciences, 28(3): 409 - 420.

Prensky, S.E., 1999. Advances in borehole imaging technology and applications. Geological Society, London, Special Publications, 159(1): 1-43.

Schepers, R., Rafat, G., Gelbke, C. and Lehmann, B., 2001. Application of borehole logging, core imaging and tomography to geotechnical exploration. International Journal of Rock Mechanics and Mining Sciences, 38(6): 867 - 876.

Thapa, B.B., Hughett, P. and Karasaki, K., 1997. Semi-automatic analysis of rock fracture orientations from borehole wall images. Geophysics, 62(1): 129-137.

Wang, C., Ge, X. and Bai, S., 2002. Study of the digital panoramic borehole camera system. Chinese Journal of Rock Mechanics and Engineering, 21(3): 398-403.

WANG, C., HU, P. and SUN, W., 2010. Method for evaluating rock mass integrity based on borehole camera technology. Rock and Soil Mechanics, 31(4): 1326-1330.

Williams, J.H. and Johnson, C.D., 2004. Acoustic and optical borehole-wall imaging for fractured-rock aquifer studies. Journal of Applied Geophysics, 55(1 - 2): 151 - 159.

Yan, C. et al., 2014. Borehole Stability in High-Temperature Formations. Rock Mechanics and Rock Engineering, 47(6): 2199-2209.

Yang, T.H. et al., 2015. Anisotropic characteristics of jointed rock mass: A case study at Shirengou iron ore mine in China. Tunnelling and Underground Space Technology, 48: 129-139.

Yang, T.H. and Chen, S.K., 2009. Coupled Model of Gas Flow -Solid Distortion in Coal Seams and Mechanisms on Pressure Relief and Gas Drainage. Flow in Porous Media - from Phenomena to Engineering and Beyond: 292-298.

Zheng, J. et al., 2014. An improved Monte Carlo simulation method for discontinuity orientations based on Fisher distribution and its program implementation. Computers and Geotechnics, 61: 266-276.

Zheng, J., Deng, J.H., Zhang, G.Q. and Yang, X.J., 2015. Validation of Monte Carlo simulation for discontinuity locations in space. Computers and Geotechnics, 67: 103-109.

Zohreh, M., Junin, R. and Jeffreys, P., 2014. Evaluate the borehole condition to reduce drilling risk and avoid potential well bore damages by using image logs. Journal of Petroleum Science and Engineering, 122: 318 - 330 . 